Pacific Journal of Mathematic 


\title{
A FINITE ALGORITHM FOR THE SOLUTION OF CONSISTENT LINEAR EQUATIONS AND INEQUALITIES AND FOR THE TCHEBYCHEFF APPROXIMATION OF INCONSISTENT LINEAR EQUATIONS
}

\author{
Allen A. Goldstein ANd Ward Cheney
}

I. Introduction. The three problems of the title are treated here from a unified geometric standpoint, and an algorithm is presented for their solution. Algorithms for these problems already exist in [8], [4], [5], and [6]. In the present algorithm, each problem is reinterpreted as one of finding the lowest points (if any exist) of a polytope in an Euclidean space; the techniques of steepest descent and elimination of variables are then combined to work downward from vertex to vertex. Professor T. S. Motzkin kindly called our attention to references [8] and [3] in which a similar viewpoint is exploited. Our thanks are also due to the referee for helpful suggestions, and to Norman Levine, who has coded the algorithm on the " 704 " automatic digital computer.

Section II which follows provides a description of the problems and an outline of the algorithm. Section III contains a detailed statement of the algorithm. Section IV concerns the special case of $n$ variables and $n+1$ equations or inequalities. Section $\mathrm{V}$ is devoted to a proof of finiteness of the algorithm. In $\S \mathrm{VI}$, relationships among the three above problems and linear programming are discussed. In particular, it is shown that a simple modification of the algorithm will permit its application to linear programming.

II. The problems defined. Let $\left\{A^{1}, \cdots, A^{m}\right\}$ be a subset of $E_{n}$ with $m \geqq n$, and let $b$ denote a fixed point of $E_{m}$. We emphasize that throughout the paper the matrix $A$ composed of the rows $A^{1}, \cdots, A^{m}$ is assumed to be of rank $n$. The notation $(u, v)$ will be used for $\sum u_{i} v_{i}$, and a vector $\bar{x}=\left(x_{1}, \cdots, x_{n+1}\right)$ will be said to have abscissa $x=\left(x_{1}, \cdots, x_{n}\right)$ and ordinate $x_{n+1}$. The word polytope denotes the intersection of a finite number of half-spaces. Define the polytope

$$
\mathscr{P}=\left\{\bar{x}: x_{n+1} \geqq \max _{1 \leqq i \leqq m}\left[\left(A^{i}, x\right)-b_{i}\right]\right\} .
$$

If $\mathscr{P}$ intersects the half-space $\left\{\bar{x}: x_{n+1} \leqq 0\right\}$, then the system

Received August 1, 1957, and in revised form March 11, 1958. Parts of this paper were presented to the American Mathematical Society as follows: June 1958 abstract 589, August 1957 abstract 748. The paper was presented by title to the Conference on Matrix Computation, Wayne State University, September 1957. 


$$
\left(A^{i}, x\right) \leqq b_{i}
$$$$
(1 \leqq i \leqq m)
$$

is consistent. Any point $\bar{x}$ of $\mathscr{P}$ lying in the aforementioned half-space has an abscissa which satisfies this system. In particular, if $\mathscr{P}$ possesses lowest points (i.e., points of minimum ordinate), then these points have this property, and their abscissae minimize the function

$$
F(x)=\max _{1 \leqq i \leqq m}\left[\left(A^{i}, x\right)-b_{i}\right] .
$$

Consider next the system of linear equations

$$
\left(A^{i}, x\right)=b_{i}
$$$$
(1 \leqq i \leqq m)
$$

The Tchebycheff problem for this system is to obtain $x$ in $E_{n}$, called a minimax solution, which minimizes the function

$$
F^{*}(x)=\max _{1 \leqq i \leqq m}\left|\left(A^{i}, x\right)-b_{i}\right| \text {. }
$$

If one defines $A^{m+i}=-A^{i}$ and $b_{m+i}=-b_{i}$ when $1 \leqq i \leqq m$, then

$$
F^{*}(x)=\max _{1 \leqq i \leqq 2 m}\left[\left(A^{i}, x\right)-b_{i}\right] .
$$

In this case another polytope may be defined, and any lowest point $\bar{x}$ will have a minimax solution $x$ for abscissa and $F^{*}(x)$ for ordinate. Note that the case of consistent linear equations is encompassed by the Tchebycheff problem, and that in the Tchebycheff problem the polytope must have a lowest point. The all-inclusive problem, then, to which attention is now turned, is that of locating the lowest points of $\mathscr{P}$ or, equivalently, of minimizing the function $F$. This unified geometric viewpoint is due to Fourier, [3].

In the simplest case, the algorithm is as follows. Define residual functions

$$
R^{i}(x)=\left(A^{i}, x\right)-b_{i} \quad(1 \leqq i \leqq m) .
$$

Then $F(x)=\max _{1 \leqq i \leqq m} R^{i}(x)$. An arbitrary initial vector $x^{9}$ will generally provide one maximum residual ; suppose $F\left(x^{0}\right)=R^{1}\left(x^{0}\right)$ for convenience. On the ray $\left\{x^{0}-t A^{1}: t \geqq 0\right\}$ let $x^{1}$ be the first point at which another residual equals $R^{1}$; suppose $F\left(x^{1}\right)=R^{1}\left(x^{1}\right)=R^{2}\left(x^{1}\right)$. The equation $R^{1}(x)=$ $R^{2}(x)$ serves to eliminate one variable from the original system, and the algorithm may begin anew in this reduced system. After at most $n-1$ eliminations and descents a point is obtained which is the abscissa of a vertex of $\mathscr{P}$. A direction for the next descent is obtained by solving a system of inequalities according to a simple prescription. The precise description of the algorithm in $\S$ III is more complicated because of certain singular cases that may arise. 
III. The algorithm. A point $x$ of $E_{n}$ is sought which minimizes the function

$$
F(x)=\max _{1 \leqq i \leqq m} R^{i}(x)=\max _{1 \leqq i \leqq m}\left[\left(A^{i}, x\right)-b_{i}\right] .
$$

The solution is obtained by repeating a cycle of steps, each of which determines a vertex of the polytope $\mathscr{P}$ at a lower level than the vertex of the preceding cycle. Since the vertices of $\mathscr{P}$ are finite in number, only one cycle need be described here. In each cycle (except the first) a point $x$ is on hand from the preceding cycle. (In the first cycle, this point is arbitrary.) It is necessary to consider certain auxiliary systems

$$
\text { (r) }\left\{\begin{array}{l}
r F(x)=\max _{i}{ }^{r} R^{i}(x) \\
{ }^{r} R^{i}(x)=\left({ }^{r} A^{i}, x\right)-{ }^{r} b_{i}
\end{array}\right.
$$

in which $x \in E_{n},{ }^{r} A^{i} \in E_{n},{ }^{r} b \in E_{m},{ }^{1} F=F,{ }^{1} R^{i}=R^{i},{ }^{1} A^{i}=A^{i},{ }^{1} b=b$, $1 \leqq r \leqq n$, and $1 \leqq i \leqq m$. A system of index $(r+1)$ is obtained from a system of index $(r)$ by the elimination of a variable via an equation of the form ${ }^{r} R^{i}(x)={ }^{r} R^{j}(x)$.

JUNCTURE I. Assume that system $(r)$ is provided and that a point $y$ in $E_{n}$ is specified. By reindexing, obtain for suitable $k$,

$$
\begin{array}{cr}
{ }^{r} F(y)={ }^{r} R^{1}(y)=\cdots={ }^{r} R^{k}(y)>{ }^{r} R^{k+i}(y) & (1 \leqq i \leqq m-k) \\
\left({ }^{r} A^{1},{ }^{r} A^{1}\right) \geqq\left({ }^{r} A^{i},{ }^{r} A^{i}\right) & (1 \leqq i \leqq k)
\end{array}
$$

If ${ }^{r} A^{1}=0$, then $r=1$ and $y$ is a solution. (See proof of Theorem J.) Otherwise, there are three cases to distinguish:

Case 1 Not all of ${ }^{r} A^{1}, \ldots,{ }^{r} A^{k}$ are multiples of ${ }^{r} A^{1}$. By rearranging, assume ${ }^{r} A^{2} \neq c^{r} A^{1}$, and set ${ }^{r} R^{1}(x)={ }^{r} R^{2}(x)$. By means of this equation, some variable, say $x_{j}$, may be eliminated from system $(r)$. The new system that arises in this way is, by definition, system $(r+1)$. The vector taken now at Juncture I (with $r+1$ replacing $r$ ) is identical with $y$, except that the $j$ th component is set equal to zero.

Case $2{ }^{r} A^{1}, \cdots,{ }^{r} A^{k}$ are all positive multiples of ${ }^{r} A^{1}$. (This includes the case $k=1$.) Let $t$ be the least positive scalar for which the point $y-t^{r} A^{1}$ induces $k+1$ or more equal maximum residuals. Define $z=y-t^{r} A^{1}$, and return to Juncture I with $z$ in place of $y$. If no such $t$ exists then $\mathscr{P}$ has no lowest point. This can happen only in the inequality problem ; any $z\left(=y-t^{r} A^{1}\right)$ for which ${ }^{r} F(z) \leqq 0$ will have for its image in the original system a solution of the inequalities.

Case 3 All of ${ }^{r} A^{1}, \cdots,{ }^{r} A^{k}$ are multiples of ${ }^{r} A^{1}$, but not all positive multiples. (This includes the case of some vanishing ${ }^{r} A^{i}$.) Proceed to Juncture II. 
JUNCTURE II. Restore those $r-1$ components of $y$ which were set equal to zero during occurrences of Case 1, obtaining thereby a vector $x$ in $E_{n}$ for which

$$
F(x)=R^{1}(x)=\cdots=R^{k}(x)>R^{k+i}(x) \quad(1 \leqq i \leqq m-k) .
$$

If further descent is possible, then the system of inequalities

$$
\left(A^{i}, d\right) \geqq 1
$$

is consistent. By Theorem $\mathrm{H}$, there exists (and is discoverable by trial) a set of $r$ indices $\left\{i_{1}, \cdots, i_{r}\right\} \subset\{1, \cdots, k\}$ such that the set $\left\{A^{i_{1}}, \cdots, A^{i} r\right\}$ is linearly independent and such that the solution $d$ of $\left(A^{i_{j}}, d\right)=1$ $(1 \leqq j \leqq r)$ satisfies (1). In the case of usual occurrence, $k=r+1$, and $d$ may be constructed as in the proof of Theorem $\mathrm{F}$. The point $v$ at which the minimum of $F$ occurs on the ray $\{x-t d: t \geqq 0\}$ is taken for the initial point of the next cycle. If no such point exists, any $t$ for which $F(x-t d) \leqq 0$ solves the inequalities.

REMARK. In the event that $k>r+1$ at Juncture II, the selection of the set of $r$ indices may be avoided by a perturbation. Of the first $k$ components of the vector $b, k-r-1$ may be increased by arbitrarily small quantities, and the algorithm continued with this perturbed system. This situation arises only a finite number of times, and a solution of a perturbed system is eventually obtained. It may be shown that if the final perturbed vector $b^{*}$ satisfies $\max \left|b_{i}-b_{i}^{*}\right|<\frac{1}{2} \delta$, where $\delta$ is the vertical separation between the two lowest vertex-bearing levels of $\mathscr{P}$, then starting with the solution of the perturbed system, the algorithm will produce in one cycle a solution of the original system. The perturbation method is well-adapted to machine computation.

IV. The case $\boldsymbol{m}=\boldsymbol{n}+\mathbf{1}$. The case $m=n+1$ of the Tchebycheff problem was considered in [6], where it was shown how the solution of an $m \times n$ system may be obtained as the solution of a certain $(n+1) \times n$ subsystem discoverable by trial. Algorithms which systematize the search for this subsystem appear in [4]. Techniques developed in this section are applicable also in the algorithm of $\S I I I$ whenever there is obtained an "ordinary" vertex of the polytope $\mathscr{P}$ (that is, a vertex involving precisely $n+1$ planes).

The norms, $N$, which are employed in this section belong to a large class of monotone norms ; i.e., they have the property that $N(u) \leqq N(v)$ whenever $\left|u_{i}\right| \leqq\left|v_{i}\right|$ for all $i$. Examples of such norms are these:

$$
\begin{aligned}
& N_{p}(x)=\left(\sum\left|x_{i}\right|^{p}\right)^{1 / p} \\
& N_{\infty}(x)=\max _{i}\left|x_{i}\right|
\end{aligned}
$$




$$
N_{w}(x)=\left(\sum x_{i}^{2}\left|w_{i}\right|\right)^{1 / 2} \quad\left(w_{i} \neq 0 \text { all } i\right) .
$$

In these terms, a well-known inequality states $(x, y) \leqq N_{1}(x) \cdot N_{\infty}(y)$. The word orthant is used for the $n$-dimensional analogue of octant. Thus, two vectors $x$ and $y$ lie in the same orthant if $\operatorname{sgn} x_{i}=\operatorname{sgn} y_{i}$ for all $i$. It is understood that $\operatorname{sgn} 0=0$.

Theorem A. Let $H$ denote a hyperplane in $E_{m}$. Let $N$ and $N^{\prime}$ denote monotone norms. The points of $H$ which minimize $N$ or $N^{\prime}$ may be taken to lie in the same orthant.

Proof. The theorem is trivial when $H$ contains the origin. In the other case, let $u$ minimize $N$ on $H$ and let $w$ minimize $N_{2}$ on $H$. Then $H=\{v:(v, w)=(w, w)\}$. By setting $u_{i}^{\prime}=u_{i} \operatorname{sgn} w_{i}$, one obtains a vector $u^{\prime}$ in the same orthant as $w$; by the monotone property of $N, N\left(u^{\prime}\right) \leqq$ $N(u)$. Since the signs of $u_{i}^{\prime}$ agree with those of $w_{i},\left(u^{\prime}, w\right) \geqq(u, w)=$ $(w, w)>0$, and the number $k=(w, w) /\left(u^{\prime}, w\right)$ is therefore well-defined. The inequality just written shows that $0<k \leqq 1$, whence $N\left(k u^{\prime}\right) \leqq N\left(u^{\prime}\right)$. Since $\left(k u^{\prime}, w\right)=(w, w), k u^{\prime}$ lies on $H$. Thus $k u^{\prime}$ minimizes $N$ on $H$. The argument may now be repeated with $N^{\prime}$ and $N_{2}$ to conclude the proof. It should be observed that the theorem would not be valid if $H$ denoted a translate of an arbitrary subspace, as for example a line in $E_{3}$. See [7].

THEOREM B. Let $H$ denote a hyperplane in $E_{m}$ not through the origin.

(1) A vector $u$ of $H$ which minimizes $N_{\infty}$ is unique if and only if the vector $w$ of $H$ which minimizes $N_{2}$ has no zero components.

(2) If the vector $w$ exhibits zero components, then there is a unique vector $u$ whose corresponding components vanish.

(3) Statements (1) and (2) remain true if $w$ is replaced by the vector $v$ which minimizes $N$ on $H, N$ being any strictly convex monotone norm.

Proof. Define $u_{i}=(w, w) \operatorname{sgn}\left(w_{i}\right) / N_{1}(w)$. Then $(u, w)=(w, w)$, whence $u$ lies on $H$. If $N_{\infty}\left(u^{\prime}\right)<N_{\infty}(u)$ then

$$
\left(u^{\prime}, w\right) \leqq N_{\infty}\left(u^{\prime}\right) \cdot N_{1}(w)<N_{\infty}(u) \cdot N_{1}(w)=(w, w)
$$

so that $u^{\prime}$ does not lie on $H$. Hence the vector $u$ defined above minimizes $N_{\infty}$ on $H$. If all components of $w$ are non-zero, then it is easy to see that any alteration of the components of $u$ will either increase $N_{\infty}(u)$ or decrease $(u, w)$, so that $u$ is unique. If $w$ has zero components, the corresponding components of $u$ are zero by the above definition. The remaining components of $u$ are uniquely determined by the same argument. Statement (3) depends on the observation that $v$ is unique, and on Theorem A. 
THeOREM C. Let $A$ denote an $(n+1) \times n$ matrix of rank $n$. Let $x$ denote the least-squares solution of $A x=b$, and let $w=A x-b$. Define $u$ as in the proof of Theorem B. Then there exists $y$ in $E_{n}$ such that $A y=$ $u+b$, and $y$ is a minimax solution of $A y=b$.

Theorem D. Let $A$ denote an $(n+1) \times n$ matrix of rank $n$. The following are equivalent when the system $A x=b$ is inconsistent:

(1) The vector $x$ which minimizes $N_{\infty}(A x-b)$ is unique.

(2) If the vector $y$ minimizes $N_{2}(A y-b)$ then $A y-b$ has no zero components.

(3) A has no singular $n \times n$ submatrices.

(4) The range of $A$ contains no coordinate axis.

Proof. (1) $\leftrightarrow$ (2) follows from Theorem B. (1) $\leftrightarrow(3)$ follows from Theorem $G$ below. (2) $\leftrightarrow(4)$ follows from the observation that $A y-b$ is normal to the range of $A$.

Theorem E. Let $A$ denote an $(n+1) \times n$ matrix of rank $n$. Let $x$ denote the least-squares solution of $A x=b$. Let $w=A x-b$. Define $N_{w}(u)=$ $\left(\sum u_{i}^{2}\left|w_{i}\right|\right)^{1 / 2}$. Let $y$ be chosen to minimize $N_{w}(A y-b)$ subject to the condition $\left(A^{i}, y\right)=b_{i}$ when $w_{i}=0$. Then $y$ is a minimax solution of $A y=b$.

Proof. The conditions on $y$ guarantee its uniqueness. From leastsquares theory,

$$
\begin{array}{cr}
\sum_{i}\left[\left(A^{i}, x\right)-b_{i}\right] A_{p}^{i}=0 & (1 \leqq p \leqq n) \\
\sum_{i}\left[\left(A^{i}, y\right)-b_{i}\right] A_{p}^{i}\left|w_{i}\right|=0 & (1 \leqq p \leqq n) .
\end{array}
$$

Now let $z$ denote a minimax solution of $A z=b$ for which $A z-b$ lies in the same orthant as $A x-b$ (Theorem A), and set $M=N_{\infty}(A z-b)$. Equation (2) remains valid when $w_{i}$ is replaced by $w_{i} / M$, and is then equivalent to the following condition on $y$ (since the indices $i$ for which $w_{i} \neq 0$ yield $\left.\left(A^{i}, z\right)-b_{i}=M\right)$ :

$$
\sum_{i}^{\prime}\left[\left(A^{i}, y\right)-b_{i}\right] A_{p}^{i}\left[\left(A^{i}, x\right)-b_{i}\right]\left[\left(A^{i}, z\right)-b_{i}\right]^{-1}=0,
$$

where $p$ runs from 1 to $n$, and where $\Sigma^{\prime}$ indicates omission of indices $i$ for which $w_{i}=0$. If $w$ has no zero components, equation (3) is satisfied if and only if $y=z$ because of (1) and the uniqueness of $y$. If $w_{i}=0$ for some $i$, then by Theorem B a minimax solution $z$ exists for which the corresponding components of $A z-b$ are zero. The remainder of the proof is then as before.

Theorem F. Let $\left\{A^{i}: 1 \leqq i \leqq n+1\right\}$ be a subset of $E_{m}$ of rank $n$. Let $u$ be chosen in $E_{n+1}$ so that 

(1) $\sum_{i=1}^{n+1} u_{i} A^{i}=0$,
(2) $u \neq 0$, and
(3) $(u, b) \geqq 0$. The system of inequalities

$$
\left(A^{i}, x^{\circ}\right) \leqq b_{i}
$$$$
(1 \leqq i \leqq n+1)
$$

is consistent if and only if either $(u, b)=0$ or $u$ exhibits a positive component.

Proof. If (4) is consistent and $(u, b)>0$, select $x$ such that $y_{i} \equiv$ $\left(A^{i}, x\right) \leqq b_{i}$. Since $(b-y, u)=(b, u)-(y, u)=(b, u)-\sum_{i}\left(A^{i}, x\right) u_{i}=(b, u)-$ $\left(x, \sum u_{i} A^{i}\right)=(b, u)>0$, it is clear that $u$ must exhibit a positive component. Next, if $(u, b)=0$, then there exists an $x$ such that $\left(A^{i}, x\right)=b_{i}$, $(1 \leqq i \leqq n+1)$. Finally, if $u$ exhibits a positive component $u_{j}$, then define $y_{i}$ as $b_{i}$ or $-\left(\sum_{k \neq j} b_{k} u_{k}\right) / u_{j}$ according to whether $i \neq j$ or $i=j$. It is quickly verified that $y_{i} \leqq b_{i}$ for all $i$, and that $(y, u)=0$. Hence there exists an $x$ such that $\left(A^{i}, x\right)=y_{i}$. This completes the proof. It is true that the set $\left\{A^{i}: i \neq j\right\}$ is linearly independent. For, if on the contrary $\sum c_{i} A^{i}=0$ with $c \neq 0$ and $c_{j}=0$, then $\sum\left(u_{i}+c_{i}\right) A^{i}=0$, contradicting the fact that $u$ is unique to within a scalar multiple. Theorems of similar nature but greater complexity can be given for the cases of more than $n+1$ inequalities.

Theorem G. Assume the minimum value of $f(x)=\max _{1 \leq i \leq n+1}\left|\left(A^{i}, x\right)-b_{i}\right|$ to be positive. Let $y$ be chosen as in Theorem $C$. For each $i$, select $u^{i}$ non-zero if possible to satisfy $\left(u^{i}, A^{j}\right)=0,(1 \leqq j \leqq n+1, j \neq i)$. Then

(1) $\left|\left(A^{j}, y\right)-b_{j}\right|$ is $f(y)$ or zero according as $u^{j}$ is or is not zero.

(2) $f(x)=f(y)$ if and only if $x$ is of the form $y+\sum^{\prime} t_{i} u^{i}$ where the summation extends over $i$ for which $u^{i} \neq 0$ and where $\left|t_{i}\right| \leqq f(y) /\left|\left(A^{i}, u^{i}\right)\right|$.

(3) The dimension of the set $S=\{x: f(x)=f(y)\}$ is equal to the number of non-zero $u^{i}$.

Proof. (1) If $u^{j} \neq 0$, then from any $x$ in $S$ one may obtain by proceeding in the direction $u^{j}$ a point $x^{\prime}$ satisfying $\left(A^{j}, x^{\prime}\right)=b_{j}$ and $\left(A^{i}, x^{\prime}\right)=$ $\left(A^{i}, x\right)$ when $i \neq j$. Thus whenever $u^{j} \neq 0,\left(A^{j}, y\right)=b_{j}$. Conversely, if $u^{j}=0$, then the set $\left\{A^{i}: i \neq j\right\}$ is of full rank. Consequently $y$ is a continuous function of $f(y)$ via Cramer's rule. If $\left|\left(A^{j}, y\right)-b_{j}\right|<f(y)$ then $f(y)$ may be decreased slightly without elevating $\left|\left(A^{j}, y\right)-b_{j}\right|$ to $f(y)$, thus contradicting the assumption that $f(y)$ be a minimum. Hence $\left|\left(A^{j}, y\right)-b_{j}\right|=f(y)$.

(2) If $x$ is of the quoted form, then a calculation shows $f(x)=f(y)$. Conversely, if $f(x)=f(y)$, then for $u^{i} \neq 0$, define $t_{i}=\left[\left(A^{i}, x\right)-b_{i}\right] /\left(A^{i}, u^{i}\right)$. Set $z=y+\Sigma^{\prime} t_{i} u^{i}$. When $u^{k} \neq 0$ then

$$
\left(A^{k}, z\right)=\left(A^{k}, y\right)+t_{k}\left(A^{k}, u^{k}\right)=b_{k}+t_{k}\left(A^{k}, u^{k}\right)=\left(A^{k}, x\right) .
$$


When $u^{k}=0$ then $\left(A^{k}, z\right)=\left(A^{k}, y\right)$, but then Cramer's rule again shows that $\left(A^{k}, y\right)=\left(A^{k}, x\right)$. Thus for all $k$ we have $\left(A^{k}, z\right)=\left(A^{k}, x\right)$, and $x=z$.

(3) This will follow from (2) if it can be shown that the non-zero $u^{i}$ are linearly independent. Supposing otherwise, we have $\Sigma^{\prime} c_{i} u^{i}=0$ where the $c_{i}$ are not all zero. Then

$$
0=\left(A^{k}, \Sigma^{\prime} c_{i} u^{i}\right)=\Sigma^{\prime} c_{i}\left(A^{k}, u^{i}\right)=c_{k}\left(A^{k}, u^{k}\right),
$$

thus showing that all $c_{k}=0$. This completes the proof.

V. Finiteness of the algorithm. We begin with the theorem $[2, p$. 104] which may be invoked at Juncture II whenever more than the usual number (which is $n+1$ ) of planes is encountered at a vertex of $\mathscr{P}$.

THEOREM H (Fan). Let the system

$$
f_{i}(x) \geqq \alpha_{i} \quad(1 \leqq i \leqq p)
$$

be consistent, where $f_{1}, \cdots, f_{p}$ are given linear functionals of rank $r$. There exist $r$ linearly independent functionals $f_{n_{1}}, \cdots, f_{n_{r}}$ among them such that every solution of the system

$$
f_{n_{k}}(x)=\alpha_{n_{k}} \quad(1 \leqq k \leqq r)
$$

is a solution of (1).

Definition. A point $x \in E_{n}$ will be called a quasi-critical point if for appropriate indices $i_{1}, \cdots, i_{k}$,

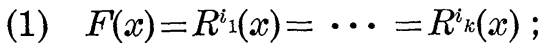

(2) the rank, $r$, of $\left\{A^{i_{1}}, \cdots, A^{i_{k}}\right\}$ is less than $k$;

(3) the system $\left(A^{i}, d\right)=1(1 \leqq j \leqq k)$ is inconsistent.

When $r=n, x$ is termed a critical point, and is the abscissa of a vertex of $\mathscr{P}$.

THEOREM I. The set of critical points is finite.

Proof. A critical point $x$, satisfying (1), (2) and (3) above, satisfies the system of equations

$$
\left(\begin{array}{ccc}
A_{1}^{i_{1}} \cdot \cdots & A_{n^{1}}^{i_{1}}-1 \\
\cdot & \cdot \cdot \cdot \cdot \\
A_{1}^{i_{k}} \cdot \cdots & \cdot \cdot A_{n}^{i_{k}}-1
\end{array}\right)\left(\begin{array}{c}
x_{1} \\
\cdot \\
\dot{x_{n}} \\
F(x)
\end{array}\right)=\left(\begin{array}{c}
b_{i_{1}} \\
\dot{\cdot} \\
\dot{b_{i k}}
\end{array}\right)
$$

where the rank of the $k$ by $n+1$ matrix is $n+1$ because of (3). Hence the solution $\left(x_{1}, \cdots, x_{n}, F(x)\right)$ is unique. Since there is only a finite number of such systems, the set of critical points is finite. 
Theorem J. At Juncture II the rank of $\left\{A^{1}, \cdots, A^{k}\right\}$ is $r$, and $r<k$.

Proof. The elimination of one component (say $\left.x_{s}\right)$ from $x=\left(x_{1}, \cdots, x_{n}\right)$ by means of an equation such as $R^{k}(x)=R^{h}(x)$ can be effected only if $A^{k}$ and $A^{h}$ are linearly independent, and leads to a new system for which the coefficient of $x_{j}$ in the $i$ th row is $A_{j}^{i}+B_{k k s j} A_{s}^{i}$ where

$$
B_{h k s j}=\left(A_{j}^{h}-A_{j}^{k}\right) /\left(A_{s}^{k}-A_{s}^{h}\right) .
$$

Thus the new matrix is obtained from the old by a sequence of elementary column operations followed by the setting of one column equal to zero. The rank of this set of rows is thus one less in the new matrix than in the old. When Juncture II arises, $r-1$ such eliminations have been made using say the residuals $R^{1}=R^{2}=\cdots=R^{r}$. Thus the rank of $A^{1}, \cdots, A^{r}$ must be $r$. Now Juncture II occurs because ${ }^{r} A^{1}$, $\cdots,{ }^{r} A^{k}$ are multiples of ${ }^{r} A^{1}$, but not all positive multiples. Not all of these are zero moreover, because if they were, the rank of the ${ }^{r-1} A^{1}$, $\cdots,{ }^{r-1} A^{k}$ would be unity and an elimination would not have been possible. Thus the rank of $A^{1}, \cdots, A^{k}$ is at most $k-1$.

THEOREM $K$. If for some set $I$ of indices there exists a $d$ in $E_{n}$ such that $\left(A^{i}, d\right)=1$ for $i$ in $I$, then $\left({ }^{r} A^{i}, d\right)=1(1 \leqq r \leqq n-1)$ whenever $i$ is in 1 .

Proof. We consider the case $r=2$. Repetitions of the argument will prove the general case. Assume $\left(A^{i}, d\right)=1$ for $i \in I$. Then for distinct $h$ and $k$ in $I$ we have $\sum_{j=1}^{n} d_{j}\left(A_{j}^{h}-A_{j}^{k}\right)=0$. Thus $d_{s}=\sum_{\substack{j \neq s \\ j=1}}^{n} d_{j} B_{h k s}$, with $B_{k k s f}$ as in the proof of Theorem J. Now for $i \in I$ we have

$$
\begin{aligned}
1 & =\sum_{j=1}^{n} A_{j}^{i} d_{j}=\sum_{\substack{j \neq s \\
j=1}}^{n} A_{j}^{i} d_{j}+A_{s}^{i} \sum_{\substack{j \neq s \\
j=1}}^{n} d_{j} B_{h k s j} \\
& =\sum_{\substack{j \neq s \\
j=1}}^{n}\left(A_{j}^{i}+A_{s}^{i} B_{h k s j}\right) d_{j}=\left({ }^{2} A^{i}, d\right) .
\end{aligned}
$$

THEOREM L. In each cycle of the algorithm, the point $x$ appearing at Juncture II is a quasi-critical point.

Proof. By Theorem J, the point $x$ satisfies criteria (1) and (2) for a quasi-critical point. Criterion (3) follows because of Theorem K. For suppose that at Juncture II the system $\left({ }^{1} A^{i}, d\right)=1$ were consistent ; then each reduced system $\left({ }^{r} A^{i}, d\right)=1$ would be consistent and Case 3 would not have arisen.

THEOREM M. To each quasi-critical point $y$ there corresponds a critical point $x$ such that $F(x)=F(y)$. 
Proof. Assume that $y$ satisfies (1), (2), and (3) in the definition of a quasi-critical point. If $r=n$, the theorem is trivial. Otherwise, select a non-zero vector $z$ orthogonal to $A^{i_{1}}, \cdots, A^{i_{k}}$. Along the ray $\{y+t z$ : $t \geqq 0\}$ the residuals $R^{i_{1}}, \cdots, R^{i_{k}}$ remain constant, and one may select the first point $y^{\prime}$ at which a new residual joins the maximum ones. This process may be repeated until a point $x$ is obtained such that for appropriate indices $i_{k+1}, \cdots, i_{h}$ we have

$$
F(x)=F(y)=R^{i_{1}}(x)=\cdots=R^{i_{h}}(x),
$$

where $h \geqq n+1$ and the rank of $\left\{A^{i_{1}}, \cdots, A^{i_{k}}\right\}$ is $n$. The system $\left(A^{i_{j}}, d\right)=1$ $(1 \leqq j \leqq h)$ is inconsistent because it has the inconsistent subsystem $\left(A^{i}, d\right)=1$ $(1 \leqq j \leqq k)$.

The following theorem includes a classical result of [1] to the effect that when each $n$ by $n$ submatrix of $A$ is non-singular then the minimax solution of $A x=b$ possesses $n+1$ equal dominating residuals.

THEOREM N. If F attains a minimum, then it does so at a critical point. Thus there are vertices among the lowest points of $\mathscr{P}$.

Proof. Let $y$ minimize $F$, and suppose that after renumbering we have $F(y)=R^{1}(y)=\cdots=R^{k}(y)>R^{k+i}(y)$. If the rank of $\left\{A^{1}, \cdots, A^{k}\right\}$ is less than $n$ then the techniques of the proof of Theorem M may be used, repeatedly if necessary, to obtain a point $x$ such that $F(y)=F(x)=$ $R^{1}(x)=\cdots R^{p}(x)>R^{p+i}(x)$ and $\left\{A^{i}: 1 \leqq i \leqq p\right\}$ is of rank $n$. If $p>n$, then $x$ is a critical point. Otherwise $x$ is a continuous function of $F(x)$ via Cramer's rule, and $F(x)$ may be decreased by so small an amount that none of $R^{p+i}(x)(1 \leqq i \leqq m-p)$ are elevated to $F(x)$-contradicting the assumption that $y$ was a solution. The proof is concluded by the observation that since $x$ is a solution, the system $\left(A^{i}, d\right)=1 \quad(1 \leqq i \leqq p)$ is inconsistent.

THEOREM O. The algorithm is finite.

Proof. In view of Theorems I, L, M, and N, it is only necessary to observe that Juncture II arises in each cycle, and that the value of $F$ is decreasing at each step.

\section{Miscellany.}

THEOREM $\mathrm{P}$. If the system of inequalities

$$
\left(A^{i}, x\right) \leqq b_{i}
$$$$
(1 \leqq i \leqq m)
$$

is consistent, then for sufficiently large $M$, every minimax solution of the system 
is a solution of (1).

Proof. Select $y$ satisfying (1). If $M \geqq-\min _{i}\left[\left(A^{i}, y\right)-b_{i}\right]$, then $\left(A^{i}, y\right)$ $-b_{\imath}+M \geqq 0$ whence $\left|\left(A^{i}, y\right)-b_{i}+M\right|=\left(A^{i}, y\right)-b_{i}+M$, and

$$
\max _{i}\left|\left(A^{i}, y\right)-b_{i}+M\right|=\max _{i}\left[\left(A^{i}, y\right)-b_{i}+M\right] \text {. }
$$

Let $x$ minimize $\max _{i}\left|\left(A^{i}, x\right)-b_{i}+M\right|$. Then

$$
\begin{aligned}
\max _{i}\left[\left(A^{i}, x\right)-b_{i}+M\right] & \leqq \max _{i}\left|\left(A^{i}, x\right)-b_{i}+M\right| \\
\leqq & \max _{i}\left|\left(A^{i}, y\right)-b_{i}+M\right|=\max _{i}\left[\left(A^{i}, y\right)-b_{i}+M\right] \leqq M .
\end{aligned}
$$

Thus $x$ satisfies (1).

REMARKS. One might expect a similar theorem to be valid for the least-squares solution of $\left(A^{i}, x\right)=b_{i}-M$. A counter-example is provided by the system

$$
2 x \leqq-1, \quad-x \leqq 1 .
$$

The least-squares solution of the system

$$
2 x=-1-M,-x=1-M
$$

is $x(M)=(-3 M-1) / 5$; but $-x(M)-1>0$ for large $M$.

THeorem Q. The following three problems are equivalent in the sense that each may be reduced to problems of the other types:

(1) The Tchebycheff problem of finding $x$ in $E_{n}$ which minimizes $\max _{1 \leqq i \leqq m}\left|\left(A^{i}, x\right)-b_{i}\right|$.

(2) The linear inequality problem of finding $x$ in $E_{n}$ such that $\left(A^{i}, x\right) \leqq b_{i},(1 \leqq i \leqq m)$.

(3) The linear programming problem of finding $x$ in $E_{n}$ which minimizes $(L, x)$ subject to $\left(A^{i}, x\right) \leqq b_{i},(1 \leqq i \leqq m)$.

Proof. That (3) subsumes (2) is trivial as $L$ may be taken arbitrarily. That (2) subsumes (3) was proved by von Neumann; see [1]. To reduce (1) to (3), double the number of $A^{i}$ as in $\S$ II. The problem is that of finding the least positive $M$ for which the system $\left(A^{i}, x\right)-M$ $\leqq b_{i}(1 \leqq i \leqq 2 m)$ is consistent, and of finding $x$ in $E_{n}$ which satisfies it. This is the same as minimizing the linear form $M$ subject to the inequalities $\left(A^{i}, x\right)-M \leqq b_{i}(1 \leqq i \leqq 2 m)$, and $-M \leqq 0$. That (1) subsumes (2) is the content of Theorem $\mathrm{P}$. This concludes the proof.

REMARK. Only small modifications of the algorithm of $\S$ III are 
required in order to apply it to linear programming. In what follows, let $\mathscr{P}$ denote the polytope $\left\{x \in E_{n}:\left(A^{i}, x\right) \geqq b_{i}\right\}$, and let $\mathscr{C}$ denote the hyperplane $\left\{x \in E_{n}:(L, x)=c\right\}$. If $(L, x)$ attains a minimum for $x$ in $\mathscr{P}$, then $c$ may be taken small enough to ensure that $\mathscr{L}$ and $\mathscr{P}$ are disjoint. (If $(L, x)$ attains a maximum on $\mathscr{P}$ then $c$ is taken large.) The point of $\mathscr{P}$ closest to $\mathscr{C}$ gives an extremum for $(L, x$.) The methods of $\S$ III may be applied first to obtain a point $x^{0}$ in $\mathscr{P}$. (Such a point is termed by some a feasible solution). One may select then the largest $t$ for which $x^{0}-t L$ still belongs to $\mathscr{P}$. There will be at least one index $i_{1}$ for which $\left(A^{i_{1}}, x^{1}\right)=b_{i_{1}}$, where $x^{1}=x^{0}-t L$. The equation $\left(A^{i_{1}}, x\right)=b_{i_{1}}$ serves to eliminate one variable from the system, and the above step is repeated in this reduced system. After at most $n-1$ eliminations and descents, a vertex $v$ of $\mathscr{P}$ is ordinarily obtained. In the usual case, $\left(A^{i_{1}}, v\right)-b_{i_{1}}$ $=\cdots=\left(A^{i} n, v\right)-b_{i_{n}}$. A direction for further descent must have the properties $\left(A^{i}, z\right) \geqq 0(1 \leqq j \leqq n)$ and $(L, z) \leqq 0$. Such a system is solvable by Theorem $\mathrm{F}$ or $\mathrm{H}$.

REMARK. The algorithm of [8], stated there for the Tchebycheff problem, is also a method which proceeds from vertex on $\mathscr{P}$. The first vertex is obtained by a process of building-up of equal maximum residuals as follows. Given a point $x$ such that $F(x)=R^{1}(x)=\cdots=R^{k}(x)$, one obtains first a vector $d$ of the form $\sum_{i=1}^{k} z_{i} A^{i}$ such that $\left(A^{1}, d\right)=\cdots$ $=\left(A^{k}, d\right)=1$. This involves solving a system of $k-1$ linear equations with $k-1$ unknowns. Along the ray $x-t d$, the first $k$ residuals remain equal to each other, and the first point at which a new residual joins the maximum ones is taken as a starting point for a repetition of the process. Assuming now that a vertex has been obtained, a test is applied to determine whether it is a lowest point of $\mathscr{P}$. This test is in essence the same as one of [6], and involves the signs of $n+1$ determinants of order $n$. If further descent is necessary, one obtains as a by-product of this test an edge issuing downward from the vertex. From this point on, only vertices are encountered, thus avoiding the above building-up process.

The method given in §III for the building-up of equal maximum residuals is more economical than that of [8]. In fact, neglecting the $n-1$ descents which both methods entail, the latter involves (besides certain matrix multiplications) the solving of $n-1$ square systems of linear equations in $2,3, \cdots, n$ variables, while the former involves roughly the labor of solving a single $n \times n$ linear system.

Starting at a vertex, it appears advisable to descend to the minimum of $F$ along whatever ray is chosen, thus presumably by-passing more vertices than when simply moving to an adjacent vertex. This of course entails repeated use of the building-up process. The following alternatives 
are offered for determining a direction for descent at a vertex.

(1) Use the method of [8].

(2) In the Tchebycheff problem, use the methods of $\S$ IV or [6] to obtain a solution of the $n \times(n+1)$ subsystem determined by those planes which enter the vertex. Move toward this point.

(3) In the inequality problem, use Theorem $\mathrm{F}$ to obtain a solution of the $n \times(n+1)$ subproblem. Move toward this point.

\section{REFERENCES}

1. George B. Dantzig, A proof of the equivalence of the programming problem and the game problem, Activity Analysis of Production and Allocation, ed. by Koopmans, New York, (1951), 330-335.

2. Ky Fan, On systems of linear inequalities, Linear Inequalities and Related Systems, ed. by Kuhn and Tucker, Princeton, (1956), 99-156.

3. J. J.-B. Fourier, Solution d'une question particulière du calcul des inéqualités, second extrait, Histoire de l'Académie des Sciences 1824, p. 48. Also available in CEUVRES DE FOURIER, II, Paris 1890, 325-328.

4. Allen A. Goldstein, James B. Herreshoff and Norman Levine, On the "best" and "least Q-th" approximaiion of an overdetermined system of linear equations, J. Assoc. Comput. Mach. 4 (1957), 341-347.

5. T. S. Motzkin and I. J. Schoenberg, The relaxation method for linear inequalities, Canad. J. Math. 6 (1954), 393-404.

6. Ch. J. de la Vallée Poussin, Sur la méthode de l'approximation minimum, Ann. Soc. Sci. Bruxelles, Seconde Partie, Mémoires 35 (1911), 1-16.

7. Problem E-1271, Amer. Math. Monthly 65 (1958), 122.

8. S. I. Zuhovicki1, An algorithm for the solution of the cebysev approximation problem in the case of a finite sysiem of incompaitible linear equations, Dokl. Akad. Nauk SSSR (N. S.) 79 (1951), 561-564, (Russian). (Translation with commentary to appear in J. Soc. Indust. Appl. Math.)

Convair Astronautics

San Diego, California 



\section{PACIFIC JOURNAL OF MATHEMATICS}

\section{EDITORS}

David Gilbarg

Stanford University

Stanford, California

R. A. Beaumont

University of Washington

Seattle 5, Washington

\author{
A. L. Whiteman
}

University of Southern California

Los Angeles 7, California

E. G. Straus

University of California

Los Angeles 24, California

\section{ASSOCIATE EDITORS}

\author{
E. F. BECKENBACH \\ C. E. BURGESS \\ M. HALL \\ E. HEWITT
}

\author{
A. HORN \\ V. GANAPATHY IYER \\ R. D. JAMES \\ M. S. KNEBELMAN
}

L. NACHBIN
I. NIVEN
T. G. OSTROM
H. L. ROYDEN

M. M. SCHIFFER

G. SZEKERES

F. WOLF

K. YOSIDA

\section{SUPPORTING INSTITUTIONS}

\author{
UNIVERSITY OF BRITISH COLUMBIA \\ CALIFORNIA INSTITUTE OF TECHNOLOGY \\ UNIVERSITY OF CALIFORNIA \\ MONTANA STATE UNIVERSITY \\ UNIVERSITY OF NEVADA \\ OREGON STATE COLLEGE \\ UNIVERSITY OF OREGON \\ UNIVERSITY OF SOUTHERN CALIFORNIA
}

\author{
STANFORD UNIVERSITY \\ UNIVERSITY OF UTAH \\ WASHINGTON STATE COLLEGE \\ UNIVERSITY OF WASHINGTON \\ AMERICAN MATHEMATICAL SOCIETY \\ CALIFORNIA RESEARCH CORPORATION \\ HUGHES AIRCRAFT COMPANY \\ THE RAMO-WOOLDRIDGE CORPORATION
}

Mathematical papers intended for publication in the Pacific Journal of Mathematics should be typewritten (double spaced), and the author should keep a complete copy. Manuscripts may be sent to any of the editors. All other communications to the editors should be addressed to the managing editor, E. G. Straus at the University of California, Los Angeles 24, California.

50 reprints per author of each article are furnished free of charge; additional copies may be obtained at cost in multiples of 50 .

The Pacific Journal of Mathematics is published quarterly, in March, June, September, and December. The price per volume (4 numbers) is $\$ 12.00$; single issues, $\$ 3.50$. Back numbers are available. Special price to individual faculty members of supporting institutions and to individual members of the American Mathematical Society: $\$ 4.00$ per volume; single issues, $\$ 1.25$.

Subscriptions, orders for back numbers, and changes of address should be sent to Pacific Journal of Mathematics, 2120 Oxford Street, Berkeley 4, California.

Printed at Kokusai Bunken Insatsusha (International Academic Printing Co., Ltd.), No. 6, 2-chome, Fujimi-cho, Chiyoda-ku, Tokyo, Japan.

\section{PUBLISHED BY PACIFIC JOURNAL OF MATHEMATICS, A NON-PROFIT CORPORATION}

The Supporting Institutions listed above contribute to the cost of publication of this Journal, but they are not owners or publishers and have no responsibility for its content or policies. 


\section{Pacific Journal of Mathematics}

\section{Vol. 8, No. 3 \\ May, 1958}

Michael Israel Aissen, A set function defined for convex plane domaines... . 383

Robert Ellis, Distal transformation groups ................... 401

Ciprian Foias, On a commutative extension of a commutative Banach algebra ....................................... 407

Jerry William Gaddum, Linear inequalities and quadratic forms ......... 411

Allen A. Goldstein and Elliott Ward Cheney, Jr., A finite algorithm for the solution of consistent linear equations and inequalities and for the Tchebycheff approximation of inconsistent linear equations...........

William L. Hart and T. S. Motzkin, Proof of the fundamental theorem on implicit functions by use of composite gradient corrections .......... 429

Henry Berge Helson, Conjugate series and a theorem of Paley .......... 437

Wu-Chung Hsiang, Abelian groups characterized by their independent subsets....................................... 447

John W. Lamperti, On the isometries of certain function-spaces ........ 459

Karel DeLeeuw and Walter Rudin, Extreme points and extremum problems

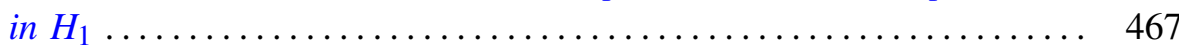

Eugene Lukacs, Some extensions of a theorem of Marcinkiewicz........ 487

George W. Mackey, Multiplicity free representations of finite groups ..... 503

Eben Matlis, Injective modules over Noetherian rings ............. 511

John William Neuberger, Continuous products and nonlinear integral equations

Lawrence Edward Payne and Hans F. Weinberger, New bounds for solutions of second order elliptic partial differential equations...

William T. Reid, A Prüfer transformation for differential systems ........ 575

Howard L. Rolf, The free lattice generated by a set of chains ...

K. M. Saksena, Inversion and representation theorems for a generalized

Laplace integral....................................... 597

Daniel Shanks, Two theorems of Gauss......................... 609

Paul Slepian, On the Lebesgue area of a doubled map ............... 613

Otto Szász and Nelson Paul Yeardley, Jr., The representation of an analytic function by general Laguerre series . ..................... 621

Alan C. Woods, On two-dimensional convex bodies ................. 635 